\title{
Fading Hawking Radiation
}

\author{
I.Sakalli. * M. Halilsoy $\dagger$ and H.Pasaoglut \\ Department of Physics, Eastern Mediterranean University, \\ G. Magusa, North Cyprus, Mersin-10, Turkey.
}

\begin{abstract}
In this study, we explore a particular type Hawking radiation which ends with zero temperature and entropy. The appropriate black holes for this purpose are the linear dilaton black holes. In addition to the black hole choice, a recent formalism in which the Parikh-Wilczek's tunneling formalism amalgamated with quantum corrections to all orders in $\hbar$ is considered. The adjustment of the coefficients of the quantum corrections plays a crucial role on this particular Hawking radiation. The obtained tunneling rate indicates that the radiation is not pure thermal anymore, and hence correlations of outgoing quanta are capable of carrying away information encoded within them. Finally, we show in detail that when the linear dilaton black hole completely evaporates through such a particular radiation, entropy of the radiation becomes identical with the entropy of the black hole, which corresponds to "no information loss".
\end{abstract}

\footnotetext{
*Electronic address: izzet.sakalli@emu.edu.tr

${ }^{\dagger}$ Electronic address: mustafa.halilsoy@emu.edu.tr

${ }^{\ddagger}$ Electronic address: hale.pasaoglu@emu.edu.tr
} 


\section{INTRODUCTION}

Hawking [12 14] and [6] showed in their seminal works that a black hole (BH) should slowly radiate away energy with its characteristic temperature and entropy. But the semiclassical picture of the Hawking radiation has a thermal nature, which poses a fundamental physical problem. Because, when the material entering the $\mathrm{BH}$ is a pure quantum state, the transformation of that state into the mixed state of Hawking radiation would destroy information about the original quantum state. However, this violates quantum mechanical unitarity and presents a physical paradox - so called the information loss paradox. For review of the topic and references on the $\mathrm{BH}$ information loss problem the reader may refer to [19, 22, 23]. There are various ideas about how the paradox could be solved. Among them, may be the most elegant and comprehensible one is the Parikh and Wilczek (PW)'s quantum tunneling formalism [20]. Their tunneling formalism is based on the null geodesics together with the WKB method. They showed explicitly how the inclusion of back-reaction effects, which guarantees the conservation of energy during a particle tunneling the horizon, yields a non-thermal correction to the $\mathrm{BH}$ radiation spectrum. For a recent review of "tunneling methods and Hawking radiation" one may consult [29]. On the other hand, the form of their non-thermal correction had a shortcoming since they did not consider the Planck-scale $(\hbar)$ quantum corrections, which elicit correlations between quanta emitted with different energies. The first attempt to fix this shortcoming came from [3], who proposed a modified version of the tunneling picture in which a leading order Planck-scale quantum correction was introduced. In addition to this, [4] have recently provided a general framework for studying quantum corrections to all orders in $\hbar$ to the entropy of a BH. When the effects of the quantum corrections are neglected, one recovers the PW's results of the BH [20]. Although there are supportive studies, see for instance [2, 5, 15, 18, 25, 32], to [4], in recent times their work has been under criticism by [34], who claimed that Banerjee and Majhi's result assumes an incorrect definition of energy. Putting aside these discussions, here we concentrate on the study which has recently been published by Singleton, Vagenas, Zhu and

Ren (SVZR) [26]. They have attempted to show that the quantum corrections to all orders in $\hbar$ can be adjusted finely so much so that both entropy and temperature of the Schwarzschild $\mathrm{BH}$ go to zero as the mass of the $\mathrm{BH}$ is radiated away, i.e. $S, T(M \rightarrow 0) \rightarrow 0$. But, immediately after it is understood that such a scenario is not possible for the Schwarzschild 
$\mathrm{BH}$ [27]. In fact, the key idea of the present study is to examine whether the quantum corrected Hawking radiation with $S, T(M \rightarrow 0) \rightarrow 0$ is possible for other types of BHs or not. In this article, we consider a general class of 4-dimensional ( $4 D)$ metric which belongs to static, spherically symmetric linear dilaton black holes (LDBHs) [10, 11] that constitute solutions to Einstein-Maxwell-Dilaton (EMD), Einstein-Yang-Mills-Dilaton (EYMD) and Einstein-Yang-Mills-Born-Infeld-Dilaton (EYMBID) theories [16]. The LDBHs are known to be a special class of non-asymptotically flat (NAF) spacetimes. The reason why we focus on the LDBHs is that by using merely the PW's quantum tunneling formalism one can not modify their thermal character of the Hawking radiation [21]. This means that the original PW's tunnelingformalism fails to answer the information loss paradox appearing in the LDBHs. Because of this, in addition to the back reaction effects we need to take into account the quantum corrections to obtain a radiation other than pure thermal [24]. As an extension of the study [24], here we consider the general form of the quantum corrected temperature given by SVZR, and apply it to the LDBHs in order to derive specific entropy and temperature, both of which go to zero with $S, T(M \rightarrow 0) \rightarrow 0$. Detailed calculations of these processes are given in the next sections, and as a result we obtain the above-mentioned radiation, and it is not pure thermal. The behaviors of both the entropy and temperature of the LDBH with the quantum correction parameters coming from String Theory (ST) and Loop Quantum Gravity (LQG) are examined. We find that the results which have no any physical ambiguity are possible only in the LQG case. Moreover, it is highlighted that higher order quantum corrections which are in conform with the back reaction effects provide the correlations between the emitted quanta. Finally, we show that the LDBHs are able to evaporate away completely with the entropy conservation (initial BH entropy is equal to the entropy of the radiation), which leads to the fact that information is not lost. Organization of the paper is as follows. In Sect. 2, we derive the entropy and temperature providing $S, T(M \rightarrow 0) \rightarrow 0$ in quantum corrected LDBHs. Sect. 3 is devoted to the entropy conservation argument and Sect. 4 completes the paper with discussion and conclusion.

Throughout the paper, the units $G=c=k_{B}=1$ and $L_{p}^{2}=\hbar$ are used. 


\section{QUANTUM CORRECTED ENTROPY AND TEMPERATURE EXPRES- SIONS FOR $4 D$-LDBHS}

As it was shown in [16], 4D-LDBHs in EMD, EYMD and EYMBID theories are described by the metric

$$
d s^{2}=-f d t^{2}+\frac{d r^{2}}{f}+R^{2} d \Omega_{2}^{2}
$$

with the metric functions

$$
f=\tilde{\Sigma}\left(r-r_{+}\right), \quad R=A \sqrt{r}
$$

It is obvious that metric (1) represents a static, non-rotating $\mathrm{BH}$ with a horizon at $r_{+}$. The dimensional constants $\tilde{\Sigma}$ and $A$ in the metric functions (2) take different values according to the concerned theory (EMD, EYMD or EYMBID) [16]. For $r_{+} \neq 0$, the horizon hides the naked singularity at $r=0$. However, in the extreme case of $r_{+}=0$, the central null singularity at $r=0$ is marginally trapped in which it does not allow outgoing signals to reach external observers. Namely, even in the extreme case of $r_{+}=0$, metric (1) maintains its BH property.

By using the definition of quasi-local mass $M$ [7] for the NAF metric (1), one finds a relation between the horizon $r_{+}$and the mass $M$ as

$$
r_{+}=\frac{4 M}{\tilde{\Sigma} A^{2}} .
$$

After some elementary dimensional analysis, one can see that the units of $M$ and $A^{2}$ are $L_{p}$, while $\tilde{\Sigma}$ has the unit of $L_{p}^{-1}$ so that $r_{+}$has the unit of $L_{p}$.

Recently, it has been shown that the temperature for a general class of static, spherically symmetric BH with quantum corrections to all orders in $\hbar$ [26] is given by

$$
T=\frac{\hbar \kappa}{2 \pi}\left(1+\sum_{j=1}^{\infty} \frac{\alpha_{j} \hbar^{j}}{r_{+}^{2 j}}\right)^{-1}
$$

where $\kappa$ is the surface gravity of the BH such that it becomes $\kappa=\frac{\tilde{\Sigma}}{2}$ for the LDBHs, and $\alpha_{j}$ 's - dimensionless constants - stand for the quantum correction terms. In this expression $\frac{\hbar \kappa}{2 \pi}$ is nothing but the well-known Hawking temperature $T_{H}$. Here, we wish to highlight one of the important features of the LDBHs that the Hawking temperature of the LDBH, 
$T_{H}=\frac{\hbar \tilde{\Sigma}}{4 \pi}$, is independent of their quasi-local mass $M$, and which is therefore a constant throughout the evaporation process i.e. an isothermal process.

In general, the first law of thermodynamics is about an expression for the entropy $(S)$ as

$$
S=\int \frac{d M}{T}
$$

where $M$ is the total energy (mass) of the BH. As we adopt the temperature with generic quantum corrections from (4), the entropy to all orders in $\hbar$ can be found by substituting (4) into (5), and by evaluating the integral. Thus, for the LDBHs one obtains the following modified entropy as a function of $M$

$$
S(M)=\frac{M}{T_{H}}\left(1-\sum_{j=1}^{\infty} \frac{\alpha_{j}}{2 j-1} x^{j}\right) .
$$

where $x=\frac{\hbar \tilde{\Sigma}^{2} A^{4}}{16 M^{2}}$ is a dimensionless quantity.

As mentioned before, our ultimate aim is to find a specific condition by which it leads to a complete radiation of the $\mathrm{LDBH}$ with $S, T(M \rightarrow 0) \rightarrow 0$. This requirement implies conditions on the $\alpha_{j}$ 's. It is remarkable to see that the only possibility which satisfies $S, T(M \rightarrow 0) \rightarrow 0$ is,

$$
\alpha_{j}=\frac{(-1)^{j+1}(2 j-1)}{j} \alpha_{1},
$$

Inserting this into the sum of (6), we find the modified LDBH entropy as

$$
S(M)=\frac{M}{T_{H}}\left[1+\alpha_{1} \ln \left(\frac{16 M^{2}}{16 M^{2}+\hbar \tilde{\Sigma}^{2} A^{4}}\right)\right],
$$

Now, it can be easily checked that $S(M \rightarrow 0) \rightarrow 0$ and $S(M \rightarrow \infty) \rightarrow \infty$. Although the result of the sum in (8) stipulates that $M>\frac{\sqrt{\hbar} \tilde{\Sigma} A^{2}}{4}$, by making an analytical extension of the zeta function [1, 26], one can redefine the sum via $\alpha_{1} \ln \left(\frac{16 M^{2}}{16 M^{2}+\hbar \tilde{\Sigma}^{2} A^{4}}\right)$ such that it becomes valid also for $M<\frac{\sqrt{\hbar} \tilde{\Sigma} A^{2}}{4}$. We plot $S(M)$ (8) versus $M$ for the cases of semi-classical and quantum corrections to all orders in $\hbar$, and display all graphs in Fig. 1. In all figures, we have used two different $\alpha_{1}$ values such that $\alpha_{1}=-\frac{1}{2}$ is taken as the representative of the LQG [17], while the choice $\alpha_{1}=\frac{1}{2}$ stands for the ST [28, 33]. Here, physically inadmissible case belongs to the ST's one in which the behavior of the entropy is not well-defined. Because, 
as seen in Fig. 1(b), just before the complete evaporation of the LDBH, the entropy first decreases to a negative value and then increases from below to become zero with $M=0$.

Furthermore, if we impose the same condition (7) in equation (4), a straightforward calculation of the sum shows that the temperature is,

$$
T(M)=\frac{T_{H}}{1+\alpha_{1}\left[\frac{2 \hbar \tilde{\Sigma}^{2} A^{4}}{16 M^{2}+\hbar \tilde{\Sigma}^{2} A^{4}}+\ln \left(\frac{16 M^{2}}{16 M^{2}+\hbar \tilde{\Sigma}^{2} A^{4}}\right)\right]} .
$$

It is obvious that removing the quantum corrections i.e., $\alpha_{1}=0$, leads $T$ to the semiclassical result, $T_{H}$. Significantly, one can easily verify that $T(M \rightarrow 0) \rightarrow 0$ and $T(M \rightarrow$ $\infty) \rightarrow T_{H}$. As it can be seen in Fig. 2(a), when $\alpha_{1}<0$ (the LQG case), the temperature does not take negative value, rather it remains always positive and goes to zero with $M \rightarrow 0$. On the other hand, for $\alpha_{1}>0$ (the ST case, see Fig. 2(b)), the temperature does not exhibit well-behaved behavior as obtained in the LQG case. Because it first diverges for some finite value of $M$, then becomes negative and approaches zero from below.

As a final remark for this section, our results suggest that the quantum corrected Hawking radiation of the LDBH should be considered with the LQG term $\alpha_{1}<0$ in order to avoid from any unphysical thermodynamical behavior. Because in the LQG case, both plots of $S(M)$ and $T(M)$ have physically acceptable thermodynamical behaviors and represent the deserved final; $S, T(M \rightarrow 0) \rightarrow 0$.

\section{ENTROPY CONSERVATION OF LDBHS IN QUANTUM CORRECTED HAWKING RADIATION}

In the WKB approximation, the tunneling rate for an outgoing positive energy particle with a field quantum of energy $\omega$, which crosses the horizon from $r_{\text {in }}(M)$ to $r_{\text {out }}(M-\omega)$, is related to the imaginary part of the particle's action $\operatorname{Im}(I)$ in accordance with

$$
\Gamma \sim e^{-2 \operatorname{Im}(I)}
$$

Here $\operatorname{Im}(I)$ is equivalent to 


$$
\begin{aligned}
\operatorname{Im}(I) & =-\frac{1}{2}[S(M-\omega)-S(M)], \\
& =-\frac{1}{2} \Delta S,
\end{aligned}
$$

which was uncovered in [20]. Let us remark that $\Delta S$ is the change in entropy of a $\mathrm{BH}$. Hence, the relationship between the tunneling rate and the entropy change satisfies

$$
\Gamma \sim e^{\Delta S}
$$

By using (8), $\Delta S$ becomes

$$
\Delta S=\frac{1}{T_{H}}\left\{-\omega+2 \alpha_{1} \ln \left[\left(\frac{M-\omega}{\hat{Y}(\omega)}\right)^{M-\omega}\left(\frac{M}{\hat{Y}(0)}\right)^{-M}\right]\right\}
$$

where

$$
\hat{Y}(\omega)=\sqrt{(M-\omega)^{2}+\frac{\hbar \tilde{\Sigma}^{2} A^{4}}{16}},
$$

After substituting (13) into (12), the tunneling rate with quantum corrections to all orders in $\hbar$ is found as

$$
\Gamma(M ; \omega)=\exp \left(-\frac{\omega}{T_{H}}\right)\left[\left(\frac{M-\omega}{\hat{Y}(\omega)}\right)^{M-\omega}\left(\frac{M}{\hat{Y}(0)}\right)^{-M}\right]^{\frac{2 \alpha_{1}}{T_{H}}}
$$

In this expression, the term $\exp \left(-\frac{\omega}{T_{H}}\right)$ arises due to the back reaction effects. The other term to the power $\frac{2 \alpha_{1}}{T_{H}}$ represents the quantum corrections to all orders in $\hbar$, and significantly it gives cause for a degeneracy in the pure thermal radiation. In the absence of the quantum corrections $\left(\alpha_{1}=0\right.$ : the semi-classical case) the radiation of the LDBH is pure thermal since the rate (15) reduces to $e^{\frac{-\omega}{T_{H}}}$. The latter case was studied in detail by [21] in which it was stated that the Hawking radiation of the LDBH leads to the information loss paradox. The essential annoyance in the pure thermal radiation is that it never allows the information transfer, which can be possible with the correlations of the outgoing radiation. So it is prerequisite to keep the quantum corrections in the tunneling rate (15) when the agenda is about obtaining a spectrum which is not pure thermal, and accordingly the correlations 
of the emitted quanta from the LDBH. In general, the statistical correlation between two successive emissions is given by [9, 30]

$$
\chi\left(\omega_{1}+\omega_{2} ; \omega_{1}, \omega_{2}\right)=\ln \left[\frac{\Gamma\left(M ; \omega_{1}+\omega_{2}\right)}{\Gamma\left(M ; \omega_{1}\right) \Gamma\left(M ; \omega_{2}\right)}\right],
$$

and from (15) and (16), one obtains the statistical correlation as

$$
\chi\left(\omega_{1}+\omega_{2} ; \omega_{1}, \omega_{2}\right)=\frac{2 \alpha_{1}}{T_{H}} \ln \left[\frac{\left(\frac{M-\omega_{1}-\omega_{2}}{\hat{Y}\left(\omega_{1}+\omega_{2}\right)}\right)^{M-\omega_{1}-\omega_{2}}}{\left(\frac{M-\omega_{1}}{\hat{Y}\left(\omega_{1}\right)}\right)^{M-\omega_{1}}\left(\frac{M-\omega_{2}}{\hat{Y}\left(\omega_{2}\right)}\right)^{M-\omega_{2}}}\right]\left(\frac{M}{\hat{Y}(0)}\right)^{M},
$$

This result shows that successive emissions are statistically dependent if and only if the quantum correction parameter $\alpha_{1}$ is non-zero. Since the amount of correlation is precisely equal to mutual information between two sequentially emitted quanta, one can deduce that the statistical correlation enables the information leakage from the LDBH during its evaporation process.

Now, one can assume that the quasilocal mass of a LDBH is a combination

on of $n$-particles with energies (masses) $\omega_{1}, \omega_{2}, \ldots \omega_{n}, M=\sum_{j=1}^{n} \omega_{j}$ in which $\omega_{j}$ is the energy of the $j^{\text {th }}$ emitted field quanta (particle). Namely, the whole radiation process constitutes of successively emitted quanta $\left(\omega_{1}, \omega_{2}, \ldots \omega_{n}\right)$ from the BH, so that the LDBH loses its mass $M$ during its evaporation, and at the final state of the evaporation we find $S, T(M \rightarrow 0) \rightarrow 0$.

The probability of a radiation composed of correlated quanta is given by the following product of the tunneling rates [30, 31]

$$
\mathrm{P}_{\mathrm{rad}}=\Gamma\left(M ; \omega_{1}\right) \times \Gamma\left(M-\omega_{1} ; \omega_{2}\right) \times \ldots \times \Gamma\left(M-\sum_{j=1}^{n-1} \omega_{j} ; \omega_{n}\right)
$$

where the probability of emission of each radiation of energy $\omega_{j}$ is given by

$$
\begin{gathered}
\Gamma\left(M ; \omega_{1}\right)=\exp \left(-\frac{\omega_{1}}{T_{H}}\right)\left\{\left[\frac{M-\omega_{1}}{Y\left(\omega_{1}\right)}\right]^{M-\omega_{1}}\left[\frac{M}{\hat{Y}(0)}\right]^{-M}\right\}^{\frac{2 \alpha_{1}}{T_{H}}}, \\
\Gamma\left(M-\omega_{1} ; \omega_{2}\right)=\exp \left(-\frac{\omega_{2}}{T_{H}}\right)\left\{\left[\frac{M-\omega_{1}-\omega_{2}}{Y\left(\omega_{2}\right)}\right]^{M-\omega_{1}-\omega_{2}}\left[\frac{M-\omega_{1}}{Y\left(\omega_{1}\right)}\right]^{-\left(M-\omega_{1}\right)}\right\}^{\frac{2 \alpha_{1}}{T_{H}}},
\end{gathered}
$$




$$
\begin{aligned}
& \Gamma\left(M-\sum_{j=1}^{n-1} \omega_{j} ; \omega_{n}\right)=\exp \left(-\frac{\omega_{n}}{T_{H}}\right)\left\{\left[\frac{M-\sum_{j=1}^{n} \omega_{j}}{Y\left(\omega_{n}\right)}\right]^{M-\sum_{j=1}^{n} \omega_{j}}\left[\frac{M-\sum_{j=1}^{n-1} \omega_{j}}{Y\left(\omega_{n-1}\right)}\right]^{-\left(M-\sum_{j=1}^{n-1} \omega_{j}\right.}\right\}^{\frac{2 \alpha_{1}}{T_{H}}}, \\
& =\exp \left(-\frac{\omega_{n}}{T_{H}}\right)\left[\frac{\omega_{n}}{Y\left(\omega_{n-1}\right)}\right]^{-\frac{2 \alpha_{1}}{T_{H}} \omega_{n}},
\end{aligned}
$$

in which

$$
Y\left(\omega_{k}\right)=\sqrt{\left(M-\sum_{j=1}^{k} \omega_{j}\right)^{2}+\frac{\hbar \tilde{\Sigma}^{2} A^{4}}{16}}
$$

Here, $\Gamma\left(M-\omega_{1}-\omega_{2}-\ldots .-\omega_{j-1} ; \omega_{j}\right)$ is the conditional probability of an emission with energy $\omega_{j}$ following the emission before the energy $\omega_{1}+\omega_{2}+\ldots+\omega_{j-1}$.

We can now substitute (19) into (18), and calculate the total probability for the whole radiation, which turns out to be

$$
\mathrm{P}_{\mathrm{rad}}=\exp \left(-\frac{M}{T_{H}}\right)\left(\frac{M}{\hat{Y}(0)}\right)^{-\frac{2 \alpha_{1} M}{T_{H}}},
$$

According to the statistical mechanics, we recall that all microstates are equally likely for an isolated system. Since the radiation of a BH can be considered as an isolated system, the number of microstates $\Omega$ in the system is $1 / \mathrm{P}_{\text {rad }}$. Thus, one calculate the entropy of the radiation $S_{\text {rad }}$ from the Boltzmann's definition as

$$
\begin{aligned}
S_{\text {rad }} & =\ln (\Omega)=\ln \left(1 / \mathrm{P}_{\mathrm{rad}}\right), \\
& =\frac{M}{T_{H}}+\frac{2 \alpha_{1} M}{T_{H}} \ln \left(\frac{M}{\hat{Y}(0)}\right), \\
& =\frac{M}{T_{H}}\left[1+\alpha_{1} \ln \left(\frac{16 M^{2}}{16 M^{2}+\hbar \tilde{\Sigma}^{2} A^{4}}\right)\right] .
\end{aligned}
$$

Clearly, the total entropy of the radiation $S_{\text {rad }}$ is equal to the entropy of the initial LDBH $S(M)(8)$. We deduce therefore that the entropy is conserved - the entropy of the original 
LDBH (before radiation, initial state) is equal to the entropy of the radiation (after radiation, final state). From the microscopic point of view of the entropy, this result shows that the number of microstates of initial and after states are same. The latter remark implies also that under specific conditions it is possible to save the information during the Hawking radiation of the LDBHs. In this way, unitarity in quantum mechanics of the Hawking radiation is also restored.

\section{DISCUSSION AND CONCLUSION}

In this article, we have used SVZR's analysis [26, 27] in order to obtain a specific radiation which yields both zero temperature and entropy for the LDBH when its mass is radiated away, i.e. $S, T(M \rightarrow 0) \rightarrow 0$. According to this analysis, the complete evaporation of a $\mathrm{BH}$ is thought as a process in which both back reaction effects and quantum corrections to all orders in $\hbar$ are taken into consideration. For this purpose, in Sect. 2 we imposed a condition on $\alpha_{j}$ 's which are the parameters of the quantum corrections to all orders in $\hbar$. Unless the quantum corrections are ignored, the choice of $\alpha_{j}$ 's works finely in the LDBHs to end up with $S, T(M \rightarrow 0) \rightarrow 0$.

Upon using the specific form of the entropy (8), we derived the tunneling rate (15) with quantum corrections to all orders in $\hbar$. Then, it is shown that this rate attributes to the correlations between the emitted quanta. On the other hand, existence of the correlations of the outgoing radiation allowed us to make calculations for the entropy conservation. Thus we proved that after a LDBH is completely exhausted due to its Hawking radiation, the entropy of the original LDBH is exactly equal to the entropy carried away by the outgoing radiation. The important aspect of this conservation is that it provides a possible resolution for the information loss paradox associated with the LDBHs. Another meaning of this conservation is that the process of the complete evaporation of the $\mathrm{LDBH}$ is unitary in regard to quantum mechanics. Because, it is precisely shown that the numbers of microstates before and after the complete evaporation are the same.

When we analyze the Figs. (1) and (2) which are about the scenario of $S, T(M \rightarrow 0) \rightarrow 0$ in the quantum corrected Hawking radiation of the $\mathrm{LDBH}$, it is seen that our specific choice

of $\alpha_{j}$ 's (7) with $\alpha_{1}=\frac{1}{2}$ from ST led to unacceptable behavior for the entropy (8) in which it gets negative values for some $M$ values. In addition to this, the behavior of the temperature 
(9) in the ST case is not well-behaved compared to the LQG case. However, we have no such unphysical thermodynamical behaviors in the LQG case. So, for the scenario of $S, T(M \rightarrow 0) \rightarrow 0$, we conclude that only the quantum correction term $\alpha_{1}$ coming from the LQG should be taken into consideration.

In conclusion, we show in detail that the scenario of $S, T(M \rightarrow 0) \rightarrow 0$ in the quantum corrected Hawking radiation is possible for the LDBHs. Furthermore, the information is conserved, and unitarity in quantum mechanics is restored in the process of complete evaporation of the LDBHs. By employing SVZR's analysis, we also confirm that quantum corrections with the back reaction effects remain crucial for the information leakage. Therefore, it should be stressed that the present study is supportive to the novel idea introduced by SVZR [26]. Finally, we point out that since the LDBHs are conformally related to the Brans-Dicke BHs [8], SVZR's analysis might work for those BHs as well.

[1] Abramowitz, M., \\& Stegun, I.A., 1965, "Handbook of Mathematical Functions", Dover, New York

[2] Akbar, M., \\& Saifullah, K., 2010, Eur. Phys. J. C. 67, 205

[3] Arzano, M., Medved, A.J.M., \\& Vagenas, E.C., 2005, J. High Energy Phys. 09, 037

[4] Banerjee, R., \\& Majhi B.R., 2008, J. High Energy Phys. 0806, 095

[5] Banerjee, R., \\& Modak, S.K., 2009, J. High Energy Phys. 0911, 073

[6] Bekenstein, J.D., 1973, \prd \7, 2333

[7] Brown, J. D. \\& York, J.W., 1993, \prd \47, 1407

[8] Cai, R.G. \\& Myung, Y.S., 1997, \prd \56, 3466

[9] Chen, Y.X. \\& Shao, K.N., 2009, Phys. Lett. B. 678, 131

[10] Cl \'\{e\}ment, G., Gal'tsov D., \\& Leygnac, C., 2003, \prd \67, 024012

[11] Cl $\backslash\{$ e $\}$ ment, G., Fabris J.C., \\& Marques, G.T., 2007, Phys. Lett. B. 651, 54

[12] Hawking, S.W., 1974, \nat \248, 30

[13] Hawking, S.W., 1975, Commun. Math. Phys. 43, 199

[14] Hawking, S.W., 1976, Commun. Math. Phys. 46, 206

[15] Majhi, B.R., 2009, \prd \79, 044005

[16] Mazharimousavi, S.H., Sakalli I., \\& Halilsoy, M., 2009, Phys. Lett. B. 672, 177 
[17] Meissner, K.A., 2004, Class. Quantum Grav. 21, 5245

[18] Mirza, B. \\& Sherkatghanad, Z., 2011, \prd \83, 104001

[19] Page, D.N., 1994, in 5th Canadian Conference on General Relativity and Relativistic Astrophysics, "Black Hole Information", eds Robert M., \\& McLenaghan R.G., World Scientific, Singapore. arXiv:9305040 [hep-th]

[20] Parikh, M.K. \\& Wilczek, F., 2000, \prl \85, 5042

[21] Pasaoglu, H. \\& Sakalli, I., 2009, Int. J. Theor. Phys. 48, 3415

[22] Preskill, J., 1992, arXiv:9209058 [hep-th]

[23] Russo, J.G., 2005, arXiv:0501132 [hep-th]

[24] Sakalli, I., Halilsoy M., \\& Pasaoglu, H., 2011, Int. J. Theor. Phys. 50, 3212

[25] Sheyki, A. \\& Teimoori, Z., 2012, Gen. Relativ. Gravit. DOI: 10.1007/s10714-012-1330-y

[26] Singleton, D., Vagenas, E.C., Zhu. T., \\& Ren, J.R., 2010, J. High Energy Phys. 08, 089

[27] Singleton, D., Vagenas, E.C., Zhu. T., \\& Ren, J.R., 2011, J. High Energy Phys. 01, 021

[28] Solodukhin, S.N., 1998, \prd 57, 2410

[29] Vanzo, L., Acquaviva, G., \\& Criscienzo, R.D., 2011, Class. Quantum Grav. 28, 183001

[30] Zhang, B., Cai Q.Y., You L., \\& Zhan, M.S., 2009, Phys. Lett. B. 675, 98

[31] Zhang, B., Cai Q.Y., Zhan, M.S., \\& You, L., 2011, Ann. Phys. 326, 350

[32] Zhu, T., Ren J.R., \\& Li, M.F., 2009, J. Cosmol. Astropart. P. 08, 010

[33] Zweibach, B., 2004, "A First Course in String Theory", Cambridge University Press, Cambridge

[34] Yale, A., 2011, Eur. Phys. J. C. 71, 1622

\section{FIGURE CAPTIONS}

Figure 1: Entropy $S(M)$ as a function of LDBH mass $M$. The relation is governed by (8). Figs. 1(a) and 1(b) stand for $\alpha_{1}=-\frac{1}{2}$ and $\alpha_{1}=\frac{1}{2}$, respectively. The two curves correspond to the semi-classical entropy (dotted curve) and entropy with quantum corrections to all orders in $\hbar$ (solid curve).

Figure 2: Temperature $T(M)$ as a function of $\mathrm{LDBH}$ mass $M$. The relation is governed by (9). Figs. 2(a) and 2(b) stand for $\alpha_{1}=-\frac{1}{2}$ and $\alpha_{1}=\frac{1}{2}$, respectively. The two curves correspond to the semi-classical temperature (dotted curve) and temperature with quantum 
corrections to all orders in $\hbar$ (solid curve). 
This figure "Fig1a.png" is available in "png" format from: http://arxiv.org/ps/1202.3259v3 
This figure "Fig1b.png" is available in "png" format from: http://arxiv.org/ps/1202.3259v3 
This figure "Fig2a.png" is available in "png" format from: http://arxiv.org/ps/1202.3259v3 
This figure "Fig2b.png" is available in "png" format from: http://arxiv.org/ps/1202.3259v3 